\title{
Vital Nodes Study on Airway Network of Silk Road Economic Belt
}

\author{
Yanbo Zhu', Hening Fan ${ }^{2}$, Guilian Feng 1 \\ ${ }^{1}$ School of Physics \& Electronic Information Engineering, Qinghai Nationalities University, Qinghai, China \\ ${ }^{2}$ School of Mathematics, Qinghai Nationalities University, Qinghai, China \\ Email: ybzhu6616@163.com
}

How to cite this paper: Zhu, Y.B., Fan, H.N. and Feng, G.L. (2017) Vital Nodes Study on Airway Network of Silk Road Economic Belt. Journal of Data Analysis and Information Processing, 5, 67-76. https://doi.org/10.4236/jdaip.2017.53005

Received: April 19, 2017

Accepted: July 10, 2017

Published: July 13, 2017

Copyright ( 92017 by authors and Scientific Research Publishing Inc. This work is licensed under the Creative Commons Attribution International License (CC BY 4.0).

http://creativecommons.org/licenses/by/4.0/

(c) (i) Open Access

\begin{abstract}
In order to study the nodes importance of the aviation network of SREB (Silk Road Economic Belt), we construct the network (ANSREB) based on Graph Theory that focused on the actually situation of civil aviation transportation of SREB. We analyzed the evaluation algorithms for nodes importance, proposed the evaluation method for nodes importance of ANSREB; the quantized values of each node (Degree, Betweennesss, Closeness) are calculated with Pajek and traffic data, and determined TOP 20 critical nodes of the network on two different conditions respectively (without and within International routes). Then we contrasted and analyzed the reason that affects the ranking of those vital nodes, which has the character of highly concentration of business and dominant status.
\end{abstract}

\section{Keywords}

Graph Theory, Silk Road Economic Belt (SREB), Node, Airway Network, Airway Network of Silk Road Economic Belt (ANSREB)

\section{Introduction}

In ancient times the Silk Road was not only a trading route, but also connected Europe civilization, East Asia civilization and Islam civilization, and built the exchange bridge of culture and economic between the West and East. The Silk Road has history for more than two thousand years and played dominant role for human progress. In order to exploit domestic advantage and improve the developing level of different area in China, the Chinese Government proposed the strategy of the Silk Road Economic Belt (SREB) and the 21st-Century Maritime Silk Road; the Belt and the Road is a national strategy and has important sense for China's modernization and leading position of the Global. The B \& R will promote the Opening and Developing of China, and promote the develop- 
ment of trade and economic with Mid Asia, West Asia, South Asia, Europe and Africa [1].

The Northwest and Southwest Area of China locates in the dominate position of the Belt; aviation transportation is an important, economical and speedy mode. After many years' construction, the two airport groups that cover these regions are taking shape, which mainly based on the Airport of Chengdu, Kunming and Chongqing of Southwest and the Airport of Xian and Urumqi of Northwest China; the cluster effect of Airports reflects gradually, which also plays an important role in promoting regional economic and social development, opening more widely to the outside world, improving urban development potential and influence. Especially the central effect of Kunming, Chengdu, Chongqing, Xian, Urumqi Airport is appearing gradually; the airports in medium and small cities play the key role in expanding the network. There are Airports like Qinghaihu, Loulan, Tazhong, Maerkang, Longnan, Baoji, Hukou, Yulin, Nujiang, etc. will come into use in the future with the National Plan for 13th Five-Year and National Civil Aviation Airport Medium and Long Term Plan carried out; the scale of the Airway Network of SREB (ANSREB) will expand; the civil aviation transportation will also play an important role in transportation network of SREB [2].

Graph Theory is widely used in various complex traffic networks as aviation network, bus network, metro network, highway and railway network etc.. Wang evaluated the evolution process of China's air transport network (ATNC) during 1952-2008 with Graph index and complex network methods, explored the allometric growth in the development history of ATNC with the fluctuating growth of nodes (cities) and edges (airlines or city pairs), of which the average path length in ATNC reduced from 5.74 in 1952 to 2.24 in 2008, the clustering coefficient rose from 0 to 0.69 [3]. Du took Shanghai metro networks as objective, create the topology of Shanghai metro network by SpaceL method, analyzed the network characteristics by calculating the characteristic indicators, which based on the complex network theory [4].

This paper aims to quantize the nodes importance of ANSREB and analyze the reasons that affect the Ranking. This paper analyzes the characteristics(degree, betweenness, closeness) of ANSREB(Domestic Part) with Pajek based on complex network and Graph Theory, calculate the quantitative value of nodes importance at different conditions, combine the key business metrics like passenger traffic and freight traffic, study the algorithms on how to determine the core nodes (airport), provide an algorithm to identify these nodes. The contrastive analyze on the Top 20 vital nodes of ANSREB are listed, the reason that affect the ranking of vital nodes are analyzed, some proposal is given for logistics, transportation, planning and related decision support departments.

This paper is organized as follow. The ANSREB based on Graph Theory is constructed in Section 2. The identification method for core nodes is listed in Section 3. The algorithm to determine the critical nodes of ANSREB is proposed also. Detailed contrastive analysis of key nodes importance is shown in Section 4. 
Finally, the conclusions and future work are given in Section 5.

\section{Construction of ANSREB}

Graph theory is widely used in various complex networks such as supply chain network, communication network, food chain network, logistics network, literature network, sensor network, Internet and so on. A realistic transportation network may be abstracted as a graph formed by nodes set $\mathrm{V}$ and edges set $\mathrm{E}$, each node in $\mathrm{V}$ represents a traffic hub, such as a railway station, an airport, a city, or a metro station, etc. Each edge in $\mathrm{E}$ denotes the line that connect two airports, two railway station, two cities or two metro stations between two nodes, so the problem of transportation network may be converted to graph issue.

After integrated analysis with the airways within SREB [2] [5] [6] [7] [8] [9], the ANSREB is constructed as Figure 1 with Pajek. Figure 1 has 184 nodes and 1232 edges. There are 73 airports (40 of Northwest, 33 of Southwest China) in Figure 1 belong to SREB, the other 111 domestic airports has direct flight or stopover airways to the 73 airports. The nodes in Figure 1 denote airport, the edges in it represent the routes that link these nodes. Each node may be identified by city name or Number. In order to adopt the network analysis, the routes data simplified as follow:

- The navigable city select as the node. If the city has 2 or more airport, the data is merged, for example, the routes of Beijing Capital Airport and Nanyuan Airport, Shanghai Hongqiao Airport and Pudong Airport, Chongqing Jiangbei Airport, Wanzhou Airport and Qianjiang Airport, Aba Hongyuan Airport and Jiuzhai Huanglong Airport, Bangkok Suvarnabhumi Airport and Don Mueang Airport are all combined.

- The network deal as a symmetric one (undirected, non-planar). If there is a route from City A to B, there is also an airway from B to A accordingly.

- Merge the direct flight and stopover routes, the stopover airways will be resolved, the route from I to $\mathrm{J}$ to $\mathrm{K}$ will be split two airways as I to $\mathrm{J}$ and $\mathrm{J}$ to $\mathrm{K}$, there will be two routes to indicate.

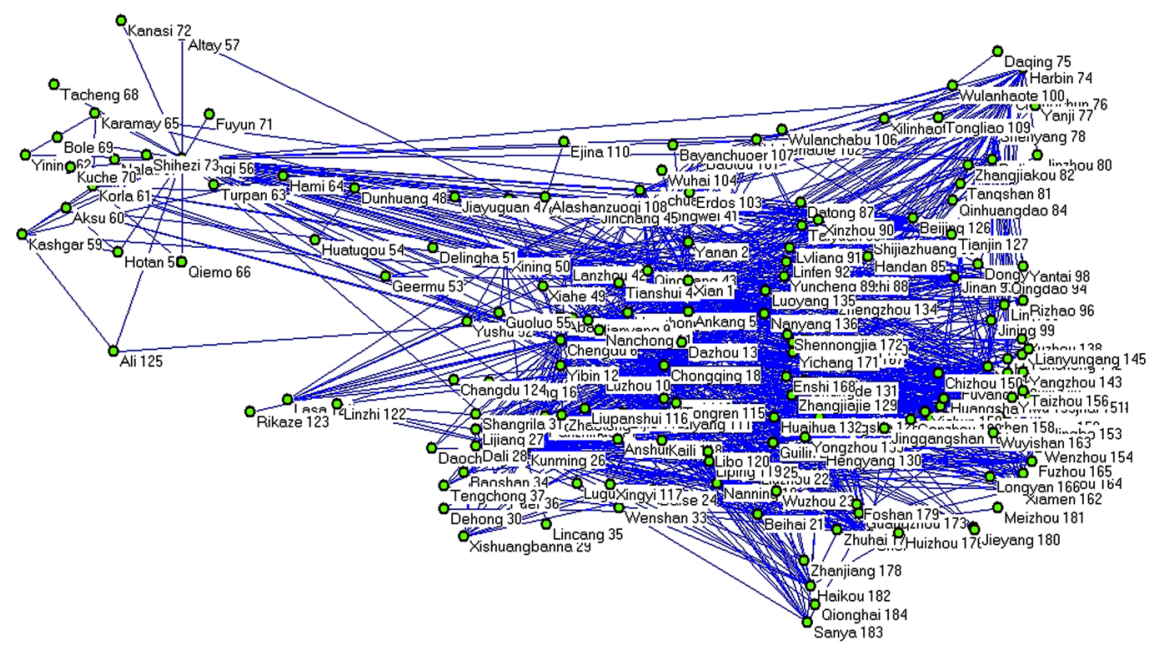

Figure 1. Airway Network of SREB(Domestic Part). 
From Figure 1 we can see that ANSREB is an unbalanced network, the routes density in east ANSREB is high while in the west is low. ANSREB is a complex network with the character of low density, key role, diversity of compose and wide coverage area. Such character matches the reality of social, population and economy developing scale of each region in SREB.

\section{Identification of Core Nodes}

The non-homogeneous topology structure of complex network decide that the importance of each key node is different, just like each person play different role in a department or team. The study on metrics and ranking of critical nodes importance on complex network is heating-up day by day, mining the nodes of various complex networks and focus on its characteristics analyze has important significance. There are many methods for identifying core nodes or evaluating its importance, such as degree, eigenvector, betweenness, subgraph, node removing, node shrinking, node cluster etc., all of which are derived from graph theory in nature.

In [10], the authors reviewed the metrics for node importance ranking in complex networks and summarized the latest progresses in this field from two prospects: the network structure and the spreading dynamics, analyzed the merits, weaknesses and applicable conditions of different node importance ranking metrics. Reference [11] proposed a multi-attribute decision-making method to identify the key nodes in complex networks. In their method, each node is regarded as a solution, and each importance evaluation criterion as one solution's attribute. Then, the closeness between each solution and the ideal solution were calculated in order to obtain the integration results of node importance in complex networks.

Reference [12] generates Hub Network of Beijing Bus, set up invulnerability index based on betweenness and degrees using complex network methods and bus smart card data. On this basis, the authors study the overall characteristics of basic-hub network. In [13] the authors propose an invulnerability evaluation model based on the number of average equivalent shortest path of the whole network. Based on the model the authors also state an evaluation method for node importance, the node is more important if the network invulnerability decreased more when the node disabled.

In [14], the authors generalize the core node mining methods in several disciplines under complex network conditions, such as social network, system science, information search, literature search etc. Some important methods of it are discussed and analyzed. Reference [15] proposes an approach based on relative entropy to evaluate the nodes importance of complex network. A system of multiple indexes is constructed firstly according to this approach. Then each evaluation result of an individual index is handled into a discrete distribution. Finally an optimal integrated evaluation solution is obtained by linear programming.

In various essential study on complex networks, mining core nodes and evaluate its importance, has important practical meaning. The value of nodes degree re- 
flect its direct influence to other nodes of the network, the closeness of nodes reflex the difficulty level reaching other nodes, which is an important index that evaluating whether the nodes in the center of the network or not, the betweenness of nodes reflect its interconnect function in the network, which is a bridge quota [16].

Passenger Traffic and Freight Traffic are the most important business metrics of civil aviation, so we choose degree, betweenness, closeness, passenger traffic and freight traffic as the index for evaluating the nodes importance of the network. As the traffic of passenger and freight are huge numbers, the degree value of each node is great than or equal to 1 , while the value of closeness and betweenness is less than 1 , there is no comparativity. So we defined five definitions of Degree Importance, Betweenness Importance, Closeness Importance, Passenger Traffic Importance and Freight Traffic Importance to normalize these values.

Definition 1. (Degree Importance): the degree importance of node i $C_{d}^{k}(i)$ defined as the degree of node $\mathrm{i}(\mathrm{d}(\mathrm{i}))$ divide the sum of all nodes degree. The formula as follow:

$$
C_{d}^{k}(i)=d(i) / \sum_{j=1}^{m} d(j)
$$

Definition 2. (Betweenness Importance): the Betweenness Importance of node i $C_{b}^{k}(i)$ defined as the betweenness of node i $C_{b}(i)$ divide the sum of all nodes betweenness. The formula as follow:

$$
C_{b}^{k}(i)=C_{b}(i) / \sum_{j=1}^{m} C_{b}(j)
$$

Definition 3. (Closeness Importance): the Closeness Importance of node i $C_{c}^{k}(i)$ defined as the degree of node i $C_{c}(i)$ divide the sum of all nodes closeness. The formula as follow:

$$
C_{c}^{k}(i)=C_{c}(i) / \sum_{j=1}^{m} C_{c}(j)
$$

Definition 4. (Passenger Traffic Importance): the Passenger Traffic Importance of node i $C_{p}^{k}(i)$ defined as the passenger traffic of node i $C_{p}(i)$ divide the sum of all nodes passenger traffic. The formula as follow:

$$
C_{p}^{k}(i)=C_{p}(i) / \sum_{j=1}^{m} C_{p}(j)
$$

Definition 5. (Freight Traffic Importance): the Freight Traffic Importance of node i $C_{f}^{k}(i)$ defined as the degree of node i $C_{f}(i)$ divide the sum of all nodes closeness. The formula as follow:

$$
C_{f}^{k}(i)=C_{f}(i) / \sum_{j=1}^{m} C_{f}(j)
$$

As the single index can not reflect the node centrality effectively, here we define the nodes importance evaluating metrics mixed by degree importance, betweenness importance, closeness importance, passenger traffic importance and freight traffic importance. The formula as follow: 


$$
C(i)=100\left(\alpha C_{d}^{k}(i)+\beta C_{b}^{k}(i)+\gamma C_{c}^{k}(i)+\lambda C_{p}^{k}(i)+\theta C_{f}^{k}(i)\right)
$$

The coefficient of $\alpha, \beta, \gamma, \lambda, \theta$ in Formula (6) satisfy the condition of $\alpha>0$, $\beta>0, \quad \gamma>0, \lambda>0, \theta>0$ and $\alpha+\beta+\gamma+\lambda+\theta=1$. As all the value of $C_{d}^{k}(i), C_{b}^{k}(i), C_{c}^{k}(i), C_{p}^{k}(i), C_{f}^{k}(i)$ are between the scale of $(0,1)$, the value of $\alpha C_{d}^{k}(i)+\beta C_{b}^{k}(i)+\gamma C_{c}^{k}(i)+\lambda C_{p}^{k}(i)+\theta C_{f}^{k}(i)$ is in $(0,1)$, so we set the value of $C(i)$ in formula 6 as $(0,100)$. The value of coefficient $\alpha, \beta, \gamma, \lambda, \theta$ can be adjusted according to the specific characteristics of real network when we evaluating the node importance of a static network, so as to reflect the relative essentiality of evaluation indicator.

Top 20 Key nodes of ANSREB is listed in Table 1, which calculate according to Figure 1 and Formula (1)-(6) separately. The meaning of Character A, B, C, $\mathrm{D}, \mathrm{E}$ represent the value of $\alpha, \beta, \gamma, \lambda, \theta$ as below:
A. $\alpha=0.2, \beta=0.2, \gamma=0.2, \lambda=0.2, \theta=0.2$
B. $\alpha=0.2, \beta=0.3, \gamma=0.1, \lambda=0.3, \theta=0.1$
C. $\alpha=0.1, \beta=0.3, \gamma=0.1, \lambda=0.3, \theta=0.2$
D. $\alpha=0.2, \beta=0.2, \gamma=0.1, \lambda=0.4, \theta=0.1$
E. $\alpha=0.2, \beta=0.2, \gamma=0.1, \lambda=0.3, \theta=0.2$

Table 1. Top 20 key nodes importance of ANSRE.

\begin{tabular}{|c|c|c|c|c|c|c|c|c|c|c|}
\hline \multirow{3}{*}{$\begin{array}{c}\text { ID } \\
1\end{array}$} & \multicolumn{2}{|l|}{ A } & \multicolumn{2}{|l|}{ B } & \multicolumn{2}{|l|}{$\mathrm{C}$} & \multicolumn{2}{|l|}{$\mathrm{D}$} & \multicolumn{2}{|l|}{$\mathrm{E}$} \\
\hline & \multicolumn{2}{|c|}{ Airport Value } & \multicolumn{2}{|c|}{ Airport Value } & \multicolumn{2}{|c|}{ Airport Value } & \multicolumn{2}{|c|}{ Airport Value } & \multicolumn{2}{|c|}{ Airport Value } \\
\hline & Chengdu & 13.27 & Chengdu & 13.17 & Chengdu & 15.75 & Chengdu & 13.68 & Chengdu & 14.97 \\
\hline 2 & Kunming & 10.92 & Xian & 12.96 & Xian & 13.60 & Xian & 12.26 & Kunming & 12.45 \\
\hline 3 & Xian & 10.67 & Kunming & 11.89 & Kunming & 13.31 & Kunming & 12.15 & Xian & 12.01 \\
\hline 4 & Chongqing & 9.23 & Chongqing & 9.65 & Chongqing & 11.01 & Chongqing & 10.11 & Chongqing & 10.53 \\
\hline 5 & Urumqi & 5.53 & Urumqi & 6.36 & Urumqi & 6.89 & Urumqi & 6.24 & Urumqi & 6.24 \\
\hline 6 & Lanzhou & 2.69 & Lanzhou & 3.07 & Lanzhou & 3.12 & Lanzhou & 3.17 & Lanzhou & 3.04 \\
\hline 7 & Nanning & 2.07 & Nanning & 2.61 & Nanning & 2.41 & Nanning & 2.84 & Nanning & 2.45 \\
\hline 8 & Guilin & 1.83 & Guilin & 2.17 & Guilin & 2.11 & Guilin & 2.14 & Guilin & 2.02 \\
\hline 9 & Yinchuan & 1.56 & Yinchuan & 1.65 & Yinchuan & 1.64 & Yinchuan & 1.79 & Yinchuan & 1.74 \\
\hline 10 & Xining & 1.38 & Xining & 1.58 & Xining & 1.57 & Xining & 1.56 & Xining & 1.50 \\
\hline 11 & Lijiang & 1.07 & Lijiang & 1.25 & Lijiang & 1.16 & Lijiang & 1.49 & Lijiang & 1.27 \\
\hline 12 & XishuangBana & 0.68 & Xishuang Bana & 0.75 & Xishuang Bana & 0.76 & Xishuang Bana & 0.91 & Xishuang Bana & 0.80 \\
\hline 13 & Mianyang & 0.66 & Mianyang & 0.67 & Mianyang & 0.57 & Mianyang & 0.74 & Mianyang & 0.69 \\
\hline 14 & Luzhou & 0.47 & Luzhou & 0.46 & Kashgar & 0.45 & Kashgar & 0.49 & Kashgar & 0.46 \\
\hline 15 & Beihai & 0.44 & Kashgar & 0.45 & Dunhuang & 0.41 & Luzhou & 0.47 & Luzhou & 0.45 \\
\hline 16 & Kashgar & 0.44 & Dunhuang & 0.44 & Korla & 0.39 & Dali & 0.46 & Beihai & 0.43 \\
\hline 17 & Dali & 0.43 & Beihai & 0.41 & Luzhou & 0.39 & Beihai & 0.45 & Dali & 0.42 \\
\hline 18 & Korla & 0.42 & Dali & 0.40 & Dali & 0.35 & Korla & 0.42 & Korla & 0.40 \\
\hline 19 & Liuzhou & 0.39 & Korla & 0.40 & Beihai & 0.35 & Yulin & 0.38 & Liuzhou & 0.37 \\
\hline 20 & Dunhuang & 0.38 & Liuzhou & 0.35 & Dehong & 0.31 & Liuzhou & 0.38 & Dunhuang & 0.35 \\
\hline
\end{tabular}


Considering the International Airways of each node in the SREB opened (there are 214 routes from the 73 nodes in SREB to the airport abroad (or outside Mainland)), add these nodes and routes to Pajek, Top $20 \mathrm{Key}$ nodes of ANSREB (Include Int. routes) list as Table 2 (The calculating method is the same as Table 1 , the meaning of Character A, B, C, D, E represent in Table 2 is also the same as Table 1 ).

\section{Key Nodes Analyze}

By contrast analyze from Table 1 and Table 2 we can see that the list of Top 13 has no difference, the sequence of Top 13 also has no change except Xian and Kunming has little difference in ranking. The Top 13 cities in Table 1 and Table 2 include 9 provincial capital (or municipality) (Chengdu, Chongqing, Kunming, Nanning, Xian, Urumqi, Lanzhou, Xining, Yinchuan), 3 National Tourist City (Guilin, Lijiang and Xishuang Bana), 1 National Science and Technology City (Mianyang), which also show the irreplaceable key role of these airports in the aviation transportation of SREB.

Table 2. Top 20 key nodes importance of ANSREB (Include international airlines).

\begin{tabular}{|c|c|c|c|c|c|c|c|c|c|c|}
\hline \multirow{3}{*}{$\begin{array}{c}\text { ID } \\
1\end{array}$} & \multicolumn{2}{|l|}{ A } & \multicolumn{2}{|l|}{$\mathrm{B}$} & \multicolumn{2}{|l|}{$\mathrm{C}$} & \multicolumn{2}{|l|}{$\mathrm{D}$} & \multicolumn{2}{|l|}{$\mathrm{E}$} \\
\hline & \multicolumn{2}{|c|}{ Airport Value } & \multicolumn{2}{|c|}{ Airport Value } & \multicolumn{2}{|c|}{ Airport Value } & \multicolumn{2}{|c|}{ Airport Value } & \multicolumn{2}{|c|}{ Airport Value } \\
\hline & Chengdu & 14.48 & Chengdu & 14.93 & Chengdu & 17.43 & Chengdu & 14.91 & Chengdu & 16.20 \\
\hline 2 & Kunming & 11.10 & Kunming & 12.18 & Kunming & 13.57 & Kunming & 12.36 & Kunming & 12.66 \\
\hline 3 & Xian & 9.35 & Xian & 11.05 & Xian & 11.71 & Xian & 10.97 & Xian & 10.71 \\
\hline 4 & Chongqing & 8.71 & Chongqing & 8.91 & Chongqing & 10.28 & Chongqing & 9.61 & Chongqing & 10.03 \\
\hline 5 & Urumqi & 6.73 & Urumqi & 8.12 & Urumqi & 8.59 & Urumqi & 7.45 & Urumqi & 7.46 \\
\hline 6 & Nanning & 2.81 & Nanning & 3.69 & Nanning & 3.43 & Nanning & 3.59 & Nanning & 3.20 \\
\hline 7 & Lanzhou & 1.54 & Lanzhou & 2.65 & Lanzhou & 2.74 & Lanzhou & 2.87 & Lanzhou & 2.74 \\
\hline 8 & Guilin & 1.42 & Guilin & 1.80 & Guilin & 1.76 & Guilin & 1.88 & Guilin & 1.75 \\
\hline 9 & Yinchuan & 1.18 & Yinchuan & 1.50 & Yinchuan & 1.52 & Yinchuan & 1.68 & Yinchuan & 1.62 \\
\hline 10 & Xining & 0.98 & Xining & 1.32 & Xining & 1.34 & Lijiang & 1.42 & Xining & 1.31 \\
\hline 11 & Lijiang & 0.63 & Lijiang & 1.17 & Lijiang & 1.09 & Xining & 1.37 & Lijiang & 1.20 \\
\hline 12 & XishuangBana & 0.59 & Xishuang Bana & 0.72 & Xishuang Bana & 0.72 & Xishuang Bana & 0.87 & Xishuang Bana & 0.76 \\
\hline 13 & Mianyang & 0.39 & Mianyang & 0.61 & Mianyang & 0.52 & Mianyang & 0.68 & Mianyang & 0.63 \\
\hline 14 & Luzhou & 0.38 & Kashgar & 0.39 & Kashgar & 0.39 & Kashgar & 0.44 & Kashgar & 0.41 \\
\hline 15 & Beihai & 0.38 & Luzhou & 0.39 & Korla & 0.34 & Dali & 0.42 & Dali & 0.39 \\
\hline 16 & Dali & 0.38 & Dali & 0.37 & Luzhou & 0.33 & Beihai & 0.41 & Beihai & 0.39 \\
\hline 17 & Kashgar & 0.35 & Beihai & 0.37 & Dali & 0.32 & Luzhou & 0.41 & Luzhou & 0.39 \\
\hline 18 & Korla & 0.34 & Korla & 0.34 & Beihai & 0.31 & Korla & 0.37 & Korla & 0.35 \\
\hline 19 & Liuzhou & 0.32 & Dunhuang & 0.32 & Dunhuang & 0.30 & Yulin & 0.36 & Liuzhou & 0.34 \\
\hline 20 & Yibin & 0.30 & Liuzhou & 0.31 & Dehong & 0.30 & Liuzhou & 0.35 & Yulin & 0.32 \\
\hline
\end{tabular}


The Top 7 airport (Chengdu, Kunming, Chongqing, Xian, Urumqi, Nanning, Lanzhou) of which Passenger Traffic over 10 million occupies 78.33 percent of the total traffic. The Top 5 airport (Chengdu, Kunming, Chongqing, Xian, Urumqi) of which Freight Traffic that over 150 thousand Ton account for 87.17 percent of the total traffic, which indicate the importance of those airport and the high concentration of business in ANSREB.

Compare with Table 1, Kunming ranking No. 2 in Table 2 under various conditions, which surpass Xian. Nanning list No. 6 exceed Lanzhou, the reason is that there are more international routes from Kunming and Nanning to Southeast Asian countries. Guilin list No. 8 in both Table 1 and Table 2, which excess Yinchuan (No. 9) and Xining (No. 10), the reason is that the betweenness of Guilin (0.0241) being great than Yinchuan (0.0095), the passenger traffic of Guilin (6340 thousand) is much larger than Xining (4340 thousand). By contrast analyze from Table 1 and Table 2 we can also find that the importance score of Chengdu, Kumimg, Urumqi and Nanning increasing, the reason is that these airports open more international airlines, the score of the other airports decreasing as these airports launch little or no Int. routes.

By contrast analyze from Table 1 and Table 2 we can also see that Chengdu, Kunming, Chongqing, Xian, Urumqi are the core nodes of SREB (The Passenger Traffic of which is great than 20 million, the importance account is great than 5.0). All the indexes of Chengdu Airport is in the lead. Kunming list in No.2, both the passenger traffic and Int. routes of which are all in No.2 also. Lanzhou, Nanning, Guilin, Yinchuan, Xining, Lijiang, Xishuang Bana are the key nodes of ANSREB, the Passenger Traffic of which is great than 4 million, the importance score of which is great than 0.59. Moreover, Mianyang, Kashgar, Luzhou, Beihai, Dali, Korla, Liuzhou, Dunhuang, Yibin, Yulin, Dehong are all significant nodes of ANSREB, the Passenger Traffic of which is great than 700 thousand, the importance score of which is great than 0.30 .

The ranking of nodes importance on ANSREB and ANSREB (include Int. airways) as show in Figure 2 and Figure 3 (The value of horizontal axis in Figure 2 and Figure 3 is the same as the nodes marked in Figure 1, the value of $\alpha, \beta$, $\gamma, \lambda, \theta$ that A, B, C, D, E taken in Figure 2 and Figure 3 is the same as in Table 1 and Table 2).

Compare Figure 2 and Figure 3 we can find that the nodes of Xian (1), Chengdu (6), Chongqing (18), Kunming (26), Urumqi (56) is towering, the nodes importance is irreplaceable, the nodes of Nanning (19), Guilin (20), Lijiang (27), Yinchuan (39), Lanzhou (42), Xining (50) is outstanding, while the other nodes is not prominent.

\section{Conclusions and Future Work}

This paper studied the nodes characteristics(degree, betweenness, closeness) of complex network on ANSREB with Pajek, combined the business data of passenger traffic and freight traffic in 2016 and the Int. routes of each airport launched in SREB, calculated the nodes importance and identified the Top 20 key nodes, and analyzed the reason that affects the ranking of those nodes. 


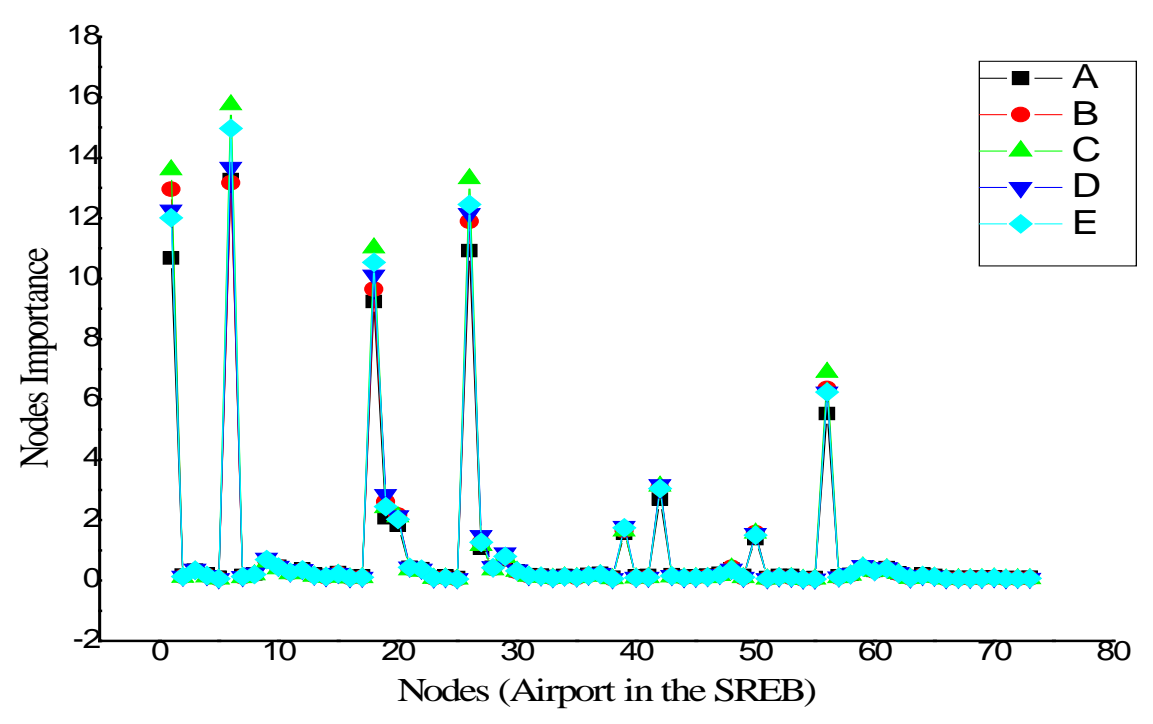

Figure 2. Nodes Importance of ANSREB.

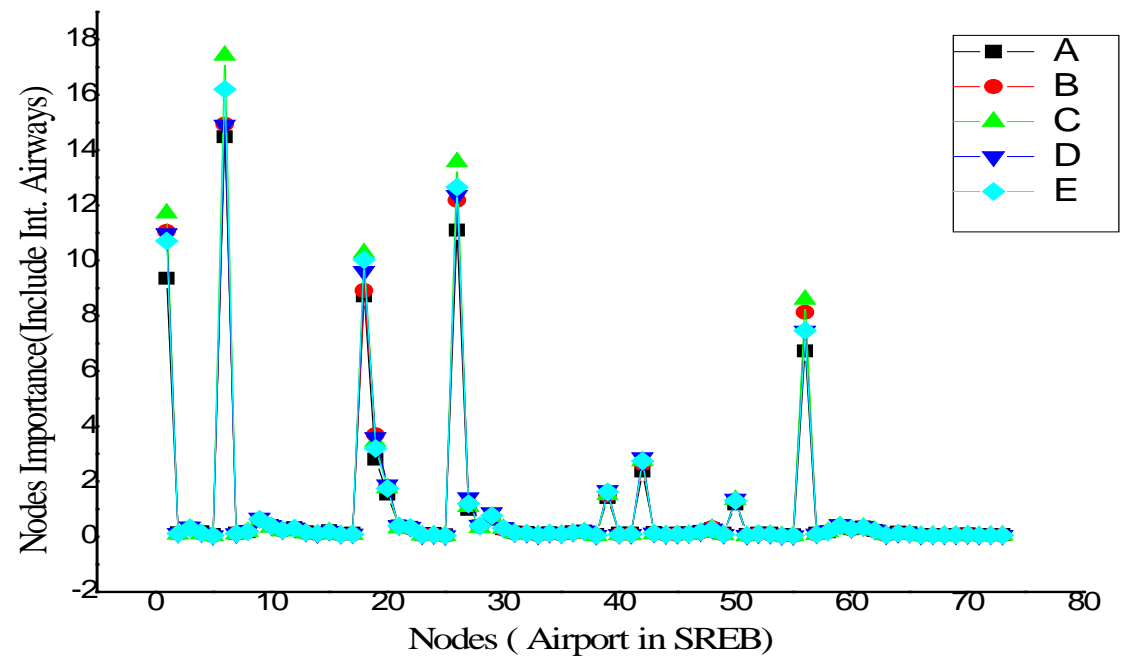

Figure 3. Nodes importance of ANSREB (Include Int. Airways).

The ANSREB (domestic part) is a complex network with the character of large scale, high concentration of business and dominant status. Chengdu, Kunming, Chongqing, Xian and Urumqi are core nodes of ANSREB, the passenger traffic, freight traffic and node importance of which are far more than the others. Lanzhou, Nanning, Guilin, Yinchuan and Xining are vital nodes, the passenger traffic, freight traffic and node importance of which are outstanding. Lijiang, Xishuang Bana, Mianyang, Kashgar, Luzhou, Beihai, Dali, Korla, Liuzhou, Dunhuang, Yibin, Yulin and Dehong are key nodes, the passenger traffic, freight traffic and node importance of which have some advantages. The other nodes are in subordinate status.

Considering the airport being built and the national medium and long term airport plan in SREB, estimating the passenger and freight traffic of each port in the future years, determining the core nodes of ANSREB will be our next-step work. 


\section{Acknowledgements}

I would like to acknowledge CAAC for providing me the Passenger Traffic and Freight Traffic of China civil aviation of 2016. My deepest gratitude goes to the Department of Science and Technology, Qinghai nationalities University for assisting me in this research. Also special thanks to Journal of Data Analysis and Information Processing for publication of this effort.

\section{References}

[1] Hu, A.G., Ma, W. and Yan, Y.L. (2014) Connotation, Definition and Passage of "Silk-Road Economic Belt" Strategy. Journal of Xinjiang Normal University (Philosophy and Social Sciences), 2, 1-10.

[2] Civil Aviation Administration of China (2017) CAAC Information Disclosure. http://www.caac.gov.cn/XXGK/XXGK/

[3] Wang, J.E. and Mo, H.H. (2014) Complex Evolution Process of China's Air Transport Network. Journal of Transportation Systems Engineering and Information Technology, 1, 71-80.

[4] Wu, J.J., Gao, Z.Y. and Sun, H.J. (2006) Complexity and Efficiency of Beijing Transit Network. International Journal of Modern Physics B, 20, 2119. https://doi.org/10.1142/S0217979206034571

[5] Xinjiang Airport Group Co., LTD. Flight Information of Urumqi Airport. http://www.xjairport.com/xjjc/

[6] Xian Xianyang International Airport (2017) Flight Information. http://www.xxia.com/site/flight/

[7] Yunnan Airport Group Co., Ltd. Flight Information of Kunming Airport. http://www.ynairport.com/FltDyn.jhtml

[8] Chendu Shuangliu International Airport. Flight Information. http://www.cdairport.com/flight/flightinfo.jsp

[9] Chongqing Airport Group Co., Ltd. Flight Information. http://www.cqa.cn/cgi/cmd/site_airinfosearch_0_0.shtml?stype $=2$

[10] Liu, J.G., Ren, Z.M. and Guo,Q. (2013) Node Importance Ranking of Complex Networks. Acta Physica Sinica, 62, Article ID: 178901.

[11] Yu, H., Liu, Z. and Li, Y.J. (2013) Key Nodes in Complex Networks Identified by Multi-Attribute Decision-Making Method. Acta Physica Sinica, 62, Article ID: 020204.

[12] Karygiannis, A., Antonakakis, E. and Apostolopoulos, A. (2006) Detecting Critical Nodes for MANET Intrusion Detection Systems. 2nd International Workshop on Security, Privacy and Trust in Pervasive and Ubiquitous Computing (SecPerU 06), Lyon, 29 June 2006, 9-15. https://doi.org/10.1109/secperu.2006.8

[13] Rao, Y.P., Lin, J.Y. and Zhou, D.F. (2009) Method for Network Invulnerability and Node Importance Evaluation. Computer Engineering, 6, 14-16.

[14] He, N., Li, D.Y. and Gan, W.Y. (2007) Mining Vital Nodes in Complex Networks. Journal of Computer Science, 12, 1-5.

[15] Chen, B., Wang, Z.X. and Luo, C. (2016) Integrated Evaluation Approach for Node Importance of Complex Networks Based on Relative Entropy. Journal of Systems Engineering and Electronics, 27, 1219-1226. https://doi.org/10.21629/JSEE.2016.06.10

[16] Huang, J.H. and Huang, F.F. (2013) 2D Index Study on Nodes Importance Evaluation of Complex Networks. Journal of Xidian University (Social Science), 5, 36-42. 
Submit or recommend next manuscript to SCIRP and we will provide best service for you:

Accepting pre-submission inquiries through Email, Facebook, LinkedIn, Twitter, etc. A wide selection of journals (inclusive of 9 subjects, more than 200 journals)

Providing 24-hour high-quality service

User-friendly online submission system

Fair and swift peer-review system

Efficient typesetting and proofreading procedure

Display of the result of downloads and visits, as well as the number of cited articles Maximum dissemination of your research work

Submit your manuscript at: http://papersubmission.scirp.org/

Or contact jdaip@scirp.org 